\title{
THE BINARITY STATUS OF STARS WITH AND WITHOUT PLANETS PROBED WITH VLT/NACO
}

\author{
S. Udry, ${ }^{1}$ A. Eggenberger, ${ }^{1}$ J.-L. Beuzit ${ }^{2}$ A.-M. Lagrange,${ }^{2}$ M. Mayor,${ }^{1}$ and G. Chauvin ${ }^{2}$
}

\begin{abstract}
RESUMEN
Las propiedades de los planetas en órbita alrededor de una componente de una estrella binaria o múltiple son distintas a las de los planetas de las estrellas aisladas (Udry et al., 2004). Describimos un sondeo con la óptica adaptativa VLT/NACO que intenta estudiar el efecto de la duplicidad estelar en la formación y evolución de los planetas, comparando la estadística de las compañeras débiles en muestras de estrellas con y sin planetas, seleccionadas en forma similar. Los resultados nos permitirán discriminar entre los modelos para la formación de planetas (acreción o inestabilidad del disco). Los resultados preliminares son muy interesantes: el $20 \pm 7 \%$ de las estrellas "sencillas" tienen compañeras dentro de $\sim 10^{\prime}$, mientras que sólo el $3 \pm 3 \%$ de compañeras equivalentes se encuentran cerca de las estrellas que albergan.
\end{abstract}

\section{ABSTRACT}

The properties of planets orbiting a component of a binary or multiple-star system appear different from the ones of planets orbiting isolated stars (Udry et al. 2004). The effect of binarity has, however, still to be better characterized and quantified. In this contribution, we describe a VLT/NACO adaptive optics survey aimed at pointing out the potential effect of stellar duplicity on planet formation and evolution, by comparing the faint-companion statistics of similarly selected subsamples of stars with and without planets. The statistical results of the survey will allow us to possibly discriminate between the proposed models of planetary formation (accretion or disk instability), some of them predicting different influences of stellar duplicity. Preliminary results are already very interesting: $20 \pm 7 \%$ of the "single" stars present companions within $\sim 10^{\prime \prime}$, whereas only $3 \pm 3 \%$ equivalent companions are found close to stars hosting planets.

Key Words: STARS: PLANETARY SYSTEMS - TECHNIQUES: HIGH ANGULAR RESOLUTION

\section{INTRODUCTION}

Among the extrasolar planets known today, 19 are orbiting a component of a binary or multiple-star system. The properties of these exoplanets (mass distribution and orbital characteristics) appear different from the ones of planets orbiting isolated stars (Zucker \& Mazeh 2002, Eggenberger, Udry \& Mayor 2004, Udry et al. 2004).

Two major scenarios have been proposed to explain giant planet formation: runaway gas accretion on a solid core or disk instability (e.g. Pollack et al. 1996; Boss 1997; Mayer et al. 2002). Interestingly, and although no consensus has been reached yet, these models might predict different influences of a stellar neighbour on planet occurrence. For example, on the one hand Boss (1998) suggests that the gravitational perturbation of the star companion

\footnotetext{
${ }^{1}$ Geneva Observatory, ch des Maillettes 51, 1290-Sauverny, Switzerland. emails: stephane.udry, anne.eggenberger, michel.mayor@obs.unige.ch

${ }^{2}$ Laboratoire d'Astrophysique, Observatoire de Grenoble, 414 rue de la piscine, St-Martin d'Hères, France. emails: JeanLuc.Beuzit, Anne-Marie.Lagrange, gael.chauvin@obs.ujfgrenoble.fr
}

may favour disk instability, and on the other hand no occurrence change is expected in the case of accretion if the disk is not too much heated by the companion.

Various other theoretical studies considering the possible influence of a stellar companion on planet formation/evolution from different approaches, including planet-core formation (disk truncation, planetesimal growth), orbital stability, and planet-disk interaction (migration), tend to conclude that stellar duplicity has a negative effect on planet formation or survival. However, the results are still preliminary, and no definite conclusion can be drawn yet.

\section{PROGRAMME AIM AND STRATEGY}

To bring more constraints to the proposed models of planetary formation, we approach the problem from an observational point of view. We have started an adaptive optics programme with NACO/VLT (Rousset et al. 2003) aiming at quantitatively characterizing the influence of stellar duplicity on planet formation and evolution.

In practice, the strategy consists of searching for (faint) companions to $\sim 110$ stars belonging to two 
different subsamples: stars with planetary companions and stars without planets. The idea is to see if, globally, some differences are observed between the two samples. For example, if the binarity rate is lower for the planet-hosting stars than for the comparison ones, then the general effect of stellar duplicity on planet formation/evolution is probably a restrictive one.

\section{SAMPLE SELECTION}

For this study, we have selected the two subsamples in the following way:

1) Planet-hosting stars: the subsample is composed of about 50 dwarf stars known to harbour extra-solar planets from the different radial-velocity surveys. They have to be observable from Paranal. We also selected only candidates that do not already appear in published equivalent $A O$ surveys.

2) Comparison stars: the subsample contains about 60 stars selected among the 1600 targets of our CORALIE planet-search programme in the southern hemisphere (Udry et al. 2000). The stars are chosen not to show "large" radial-velocity variations. They also have not to be known as Hipparcos close visual binaries (usually discarded from the radial-velocity surveys searching for exoplanets). These criteria insure a selection matching the selection of the planethosting star sample.

The 60 comparison stars have also been carefully chosen so they can be used both as references for the statistical analysis and as point spread function (PSF) reference stars in the reduction process (PSF deconvolution). To achieve this goal, the comparison-star $\alpha, \delta, V, B-V$ (and $\pi$ if possible) parameters are chosen close to the actual corresponding values of one of the planet-hosting stars. We cannot exclude, of course, that some of the comparison stars will turn out, in the future, to be in reality planet-hosting stars. This is partly the reason why the comparison star sample is larger than the planethosting star one. The set of known exoplanets will also enlarge in the future.

Finally, as a by-product, this programme may also reveal new stellar systems hosting planets, allowing us to further delimit the emerging characteristics of planets in binaries (Udry et al. 2004).

\section{OBSERVATIONS AND DETECTION LIMITS}

Up to now, five nights have been granted to this survey. The programme is thus about mid course. The observational strategy consists of observing in succession each component of a pair planetcomparison stars, in narrow-band filters within the
$\mathrm{H}$ or $\mathrm{K}$ bands, depending on the meteorological conditions. Proceeding in this way, the data obtained for one star can be used as an estimate of the PSF for the other star (at least as long as no close companion is present around any of the two stars). Whenever a companion is detected, a supplementary observation is made in $\mathrm{J}$ for better resolution and in order to obtain a colour index.

Given our exposure times and experimental settings, the detection limits $(5 \sigma)$ in $\mathrm{H}\left(\lambda_{c}=1.644 \mu \mathrm{m}\right)$ are $\Delta H \sim 3.2$ at $0.1^{\prime \prime}$ and $\Delta H \sim 9$ for $0.7^{\prime \prime} \lesssim \rho \lesssim$ $3.3^{\prime \prime}$. In $\mathrm{K}\left(\lambda_{c}=2.166 \mu \mathrm{m}\right)$, the detection limits $(5 \sigma)$ are $\Delta K \sim 2.5$ at $0.1^{\prime \prime}$ and $\Delta K \sim 7$ for $0.6^{\prime \prime} \lesssim \rho \lesssim 9^{\prime \prime}$. Most of our stars being at a distance between 10 and $100 \mathrm{pc}$, our survey will probe systems with projected separations from a few AU up to about $1000 \mathrm{AU}$.

\section{PRELIMINARY RESULTS}

Until now, 28 stars hosting planets have been observed and 1 companion has been found. The binarity rate for the planet-hosting star sample is thus $3 \pm 3 \%$. For the comparison star sample, we have observed 34 stars and found 7 companions. The binarity rate is therefore $20 \pm 7 \%$. Error bars have been computed using the bootstrap method. On the basis of these preliminary results, we find a significant difference between the two subsamples which indicates that planets are more likely to form around single stars than around the components of binary or multiple-star systems. Globally, stellar multiplicity seems thus to have a negative effect on planet formation and/or survival.

This resuit is, however, based on small samples and we will have to wait for the whole survey to be completed before reaching a statistically significant and definitive conclusion. We also need secondepoch observations in order to confirm that the detected companions are physical and not optical.

\section{REFERENCES}

Boss, A.P. 1997, Science, 276, 1836

Boss, A.P. 1998, BAAS, 30, 1057

Eggenberger, A., Udry, S., \& Mayor, M. 2004, A\&A, in press

Mayer, L., Quinn, T., Wadsley, J., \& Stadel, J. 2002, Science, 298, 1756

Pollack, J. B., Hubickyj, O., Bodenheimer, P., Lissauer, J. J., Podolak, M., \& Greenzweig, Y. 1996, Icarus, 124,62

Rousset, G. et al., 2003, SPIE 4839, 140

Udry, S., Eggenberger, A., Mayor, M., Mazeh, T., \& Zucker, S. 2004, this volume

Udry, S., Mayor, M., Naef, D.. Pepe, F., Queloz, D., Santos, N.C. Burnet, M., Confino, B., \& Melo, C. 2000, $\mathrm{A} \& \mathrm{~A}, 356,590$

Zucker, S., \& Mazeh, T. 2002, ApJ, 568, L113 